\title{
Eficacia del ordeño del cordón umbilical comparado con el pinzamiento temprano para prevenir la anemia en recién nacidos prematuros
}

\section{Efficacy of umbilical cord milking compared with early clamping to prevent anemia in preterm newborns}

\author{
Henry Sergio Carrillo-Arteaga, ${ }^{1}$ Sandy Ureña-Martínez, ${ }^{2}$ Genaro Isidoro-Jiménez, ${ }^{3}$ Nancy Leticia Villasana- \\ Carrillo $^{4}$
}

\section{Resumen}

OBJETIVO: Evaluar la eficacia del ordeño del cordón umbilical comparado con el pinzamiento temprano para prevenir la anemia en niños prematuros.

MATERIALES Y MÉTODOS: Ensayo clínico abierto, con asignación al azar a uno de dos grupos: pinzamiento temprano y ordeño de cordón umbilical. Parámetros de estudio: evaluación de la hemoglobina basal, requerimiento y cantidad de transfusiones, volumen sanguíneo total transfundido y frecuencia de reacciones adversas. Para la comparación de variables continuas se utilizó t de Student, U de Mann Whitney para variables no paramétricas y $\chi^{2}$ para variables categóricas, además del análisis multivariado corregido para trasfusión ajustada al peso al nacer, comorbilidades, hemoglobina basal y tipo de procedimiento. Se consideró significativa la $\mathrm{p}<0.05$.

RESULTADOS: Se estudiaron 91 pacientes, 46 (50.5\%) en el grupo con ordeño y 45 $(49.5 \%)$ en el grupo con pinzamiento temprano. La hemoglobina a las 24 horas de vida en el grupo con ordeño fue significativamente mayor: $17.08 \pm 2 \mathrm{~g} / \mathrm{dL}$ vs $14.74 \pm$ $1,78 \mathrm{~g} / \mathrm{dL} ; \mathrm{p}=0.001$. La frecuencia de anemia: $22(47.8 \%)$ vs $33(73.3 \%) ; \mathrm{p}=0.001$, el requerimiento de transfusión:19 (41.3\%) vs $31(68.8 \%) ; p=0.002$, y el volumen medio transfundido: $18.2 \pm 33.6 \mathrm{~mL}$ vs $35.3 \pm 45 \mathrm{~L} ; \mathrm{p}=0.04$ fueron significativamente menores en el grupo con ordeño al compararlo con el pinzamiento temprano. No hubo diferencias en policitemia $(p=0.57)$; hiperbilirrubinemia $(p=0.81)$ y estancia hospitalaria $(p=0.21)$. La concentración de hemoglobina basal, el peso menor de 1000 gramos, la edad gestacional menor de 30 semanas y el pinzamiento temprano se relacionaron directamente con la necesidad de transfusión.

CONCLUSIONES: El ordeño del cordón umbilical es un procedimiento efectivo y seguro para prevenir la anemia en los recién nacidos prematuros.

PALABRAS CLAVE: Cordón umbilical; recién nacido; policitemia; anemia; hemoglobina; comorbilidades; volumen sanguíneo.

\section{Abstract}

OBJECTIVE: To know the efficacy of milking the umbilical cord compared with early clamping to prevent anemia in premature infants.

MATERIAL AND METHODS: Open randomized clinical trial in two groups: early clamping or umbilical cord milking. We evaluate basal hemoglobin, requirement and number of transfusions, total blood volume transfused and frequency of adverse reactions. We used T Student to compare continuous variables, $U$ de Mann Whitney to parametric variables and $\chi^{2}$ to categorical variables, in addition we perform corrected multivariate analyses for transfusion adjusted to birth weight, comorbidities, basal hemoglobin and type of procedure. It was considered significant $p<0.05$.
${ }^{1}$ Pediatra-neonatólogo, maestro en Salud Pública y Administración de hospitales, adscrito al servicio de Neonatología. ${ }^{2}$ Médico residente de Neonatología.

${ }_{3}^{3}$ Pediatra-neonatólogo, adscrito al servicio de Neonatología.

${ }^{4}$ Médica cirujana y partera, maestra en ciencias, jefa del servicio de Consulta externa.

Hospital de Especialidades de la Ciudad de México Dr. Belisario Domínguez, Secretaría de Salud de la Ciudad de México

Recibido: 23 de mayo 2017

Aceptado: 14 de marzo 2019

Correspondencia Henry Sergio Carrillo Arteaga. sercarrillo@yahoo.com.

Este artículo debe citarse como Carrillo Arteaga HS, Ureña Martínez S, Isidoro Jiménez G, Villasana Carrillo NL. Eficacia del ordeño del cordón umbilical comparado con el pinzamiento temprano para prevenir la anemia en recién nacidos prematuros. Acta Pediatr Mex. 2019;40(3):122-132. 
RESULTS: We studied 91 premature patients, $46(50.5 \%)$ in the milking group and 45 $(49.5 \%)$ in the group with early clamping. Hemoglobin at 24 hours of age in the milking group was significantly higher: $17.08 \pm 2 \mathrm{~g} / \mathrm{dL}$ vs $14.74 \pm 1,78 \mathrm{~g} / \mathrm{dL} ; \mathrm{p}=0.001$. The frequency of anemia: $22(47.8 \%)$ vs $33(73.3 \%) ; p=0.001$, the transfusion requirement: $19(41.3 \%)$ vs $31(68.8 \%) ; p=0.002$, and the mean volume transfused: $18.2 \pm 33.6 \mathrm{~mL}$ vs $35.3 \pm 45 \mathrm{~mL} ; \mathrm{p}=0.04$ were significantly lower in the milking group compared with the early clamping group. There were no differences in polycythemia presentation ( $p$ $=0.57)$; hyperbilirubinemia $(p=0.81)$ and hospital stay $(p=0.21)$. The level of basal hemoglobin, weight $<1000 \mathrm{~g}$, gestational age $<30$ weeks and early clamping was directly related to the need for transfusion.

CONCLUSIONS: The milking of the umbilical cord is a useful and safe procedure for the prevention of anemia in premature newborns.

KEY WORDS: Umbilical cord; Infant, Newborn; Polycythemia; Anemia; Hemoglobins; Comorbidity; Blood Volume.

\section{ANTECEDENTES}

La anemia es uno de los padecimientos más frecuentes en los recién nacidos prematuros y por sí misma es complicada y motivo de retraso en el alta hospitalaria. ${ }^{1,2}$ Esta afección tiene una frecuencia inversamente proporcional a la edad gestacional, con límites entre 65 y 95\% de los pacientes prematuros y es más frecuente en los recién nacidos con edad gestacional menor a 32 semanas. $^{3}$

Las principales causas de anemia en este grupo incluyen: ${ }^{4,5}$

a. Inadecuada producción de eritrocitos por eritropoyesis ineficaz, debido a la producción insuficiente de eritropoyetina.

b. Acortamiento de la vida media o hemolisis. La vida media del eritrocito en el recién nacido pretérmino se reduce en 35 a 50 días, quizá debido a las menores concentraciones de ATP intracelular y de carnitina, menor actividad enzimática, mayor susceptibilidad a la oxidación lipídica y a la fragmentación de la membrana, a diferencia de lo que ocurre en el recién nacido a término en el que tiene una vida media de 90 días. c. Pérdidas sanguíneas: por transfusión fetomaterna, fetoplacentaria, gemelo a gemelo y extracciones repetidas. Estas últimas son una causa importante de anemia en los prematuros con peso muy bajo al nacer, en quienes la extracción de 1\% de su volemia por día (casi siempre para realizar exámenes de laboratorio) puede incrementar las necesidades de transfusión sanguínea hasta en $50 \%$ en las primeras dos semanas de vida extrauterina. Otras causas de pérdida sanguínea incluyen: hemorragias internas, cefalohematoma, suprarrenal, intraventricular y subgaleal. ${ }^{5}$

En la actualidad, la aplicación profiláctica de eritropoyetina humana a los prematuros permite disminuir este padecimiento, aunque la transfusión sanguínea es aún el procedimiento más efectivo y más utilizado para su tratamiento. Es importante reconocer que se trata de un procedimiento temporal e imperfecto, no exento de riesgos. ${ }^{6,7}$ Por esta razón, el ordeño del cordón umbilical puede prevenir el padecimiento y sus complicaciones, al favorecer la transferencia de un volumen de sangre considerablemente mayor en relación con el que se transfiere cuando se efectúa el pinzamiento temprano del cordón. ${ }^{8}$ 
El ordeño del cordón umbilical es un procedimiento sencillo que consiste en la transferencia activa de sangre desde el cordón hacia el recién nacido. ${ }^{8,9}$ Inicialmente, el obstetra es quien efectúa dos ordeños en dirección de la placenta al recién nacido y, posteriormente, lleva a cabo el pinzamiento del cordón dejando un segmento no menor a $20 \mathrm{~cm}$ (con lo que se asegura que se transfieran de 1 a $1.5 \mathrm{~mL}$ de sangre por centímetro de cordón umbilical). Con este segmento de cordón otro médico debidamente adiestrado realiza tres ordeños sucesivos en dirección al recién nacido. Este procedimiento puede tener efectos similares al pinzamiento tardío del cordón umbilical en prevención de anemia, reducción de hemorragia intraventricular, sepsis, enterocolitis necrosante, retinopatía del prematuro y displasia broncopulmonar. ${ }^{10-14}$ A diferencia del pinzamiento tardío, el ordeño del cordón no retrasa el proceso de reanimación neonatal, sobre todo en prematuros críticamente enfermos, lo que puede constituir una ventaja para este procedimiento. ${ }^{15}$

El pinzamiento temprano del cordón umbilical; es decir, antes de los 30 segundos de vida, aún es una práctica habitual en nuestro medio asociada con una serie de complicaciones en el recién nacido, incluida la mayor frecuencia de anemia. ${ }^{16,17}$

El objetivo de este estudio consistió en evaluar la eficacia del ordeño del cordón umbilical comparada con el pinzamiento temprano para prevenir la anemia en niños prematuros.

\section{MATERIALES Y MÉTODOS}

Ensayo clínico abierto, con asignación al azar, efectuado en dos grupos de recién nacidos (pinzamiento temprano y ordeño de cordón umbilical) atendidos entre el 14 de mayo de 2015 y el 14 de mayo de 2016 en el Hospital de Especialidades de la Ciudad de México Dr. Belisario Domínguez. Criterio de inclusión: recién nacidos menores de 37 semanas de gestación. Criterios de exclusión: malformaciones congénitas mayores, hijos de madres con isoinmunización a Rh conocida, hijo de madre con VIH, hidrops fetal y desprendimiento de placenta. Criterios de eliminación: cordón umbilical corto (menos de 20 $\mathrm{cm}$ ), vasos umbilicales muy delgados (escaso volumen sanguíneo), pacientes que no ingresaron a la unidad de cuidados intensivos neonatales, trasladados a otros centros hospitalarios o que fallecieron durante su estancia en el servicio.

\section{Procedimiento}

Los obstetras, neonatólogos y residentes de neonatología que tenían a su cargo la atención inicial del recién nacido recibieron adiestramiento específico para llevar a cabo el procedimiento en estudio mediante la proyección de videos explicativos. Posteriormente, mediante la ejecución, al azar, del procedimiento en pacientes. Quedó claro que los obstetras estaban capacitados cuando efectuaban adecuadamente dos ordeños en dirección de la placenta al recién nacido y que pinzaban el cordón umbilical dejando un segmento no menor a $20 \mathrm{~cm}$. Los neonatólogos y residentes de neonatología podían realizar adecuadamente tres ordeños sucesivos (para transferir el volumen de sangre que quedaba en el cordón) en dirección al recién nacido. En ningún caso la reanimación neonatal básica o avanzada debería interrumpirse por el procedimiento, pues éste puede efectuarse en forma simultánea.

El pinzamiento temprano del cordón umbilical sigue siendo una práctica clínica habitual en los médicos obstetras de nuestro hospital, de modo que en este caso no se requirió capacitación.

El reclutamiento de la madre y su hijo se efectúo con el diagnóstico prenatal de edad gestacional menor a 37 semanas (por ultrasonido obstétrico y clínica), establecido por el obstetra al 
ingreso de la paciente al servicio de Urgencias Tocoquirúrgicas. La madre, de quien se solicitó el consentimiento informado al ingreso, desconocía el tipo de procedimiento a efectuar en el recién nacido. Enseguida de la selección inicial, una persona ajena al servicio efectuó la asignación al azar mediante un generador computarizado de números aleatorios (java.util. Random) para participar en el grupo con ordeño o con pinzamiento temprano del cordón.

La edad gestacional del recién nacido se corroboró mediante una rápida exploración física efectuada por el neonatólogo. Enseguida, el recién nacido se asignaba para participar en uno u otro grupo y se efectuaba el seguimiento de éste durante toda su estancia hospitalaria. Solo un médico neonatólogo, exclusivamente asignado al área tocoquirúrgica, conocía el tipo de procedimiento llevado a cabo en los pacientes porque el seguimiento en la unidad de cuidados intensivos lo hizo otro personal que desconocía por completo si el recién nacido había recibido ordeño o pinzamiento temprano.

\section{Seguimiento}

En la unidad de cuidados intensivos se registraron los datos generales, el diagnóstico principal de ingreso y durante toda la estancia hospitalaria, el seguimiento clínico y los reportes de laboratorio: concentraciones de hemoglobina basal a las 24 horas de vida, diagnóstico de anemia (concentraciones de hemoglobina menores de 2 desviaciones estándar para la edad gestacional) ${ }^{18}$ requerimiento de transfusión por anemia, cantidad de transfusiones sanguíneas, volumen total de transfusión, diagnóstico de policitemia (hematocrito mayor de 65\%), hiperbilirrubinemia (concentraciones de bilirrubina por arriba de la percentila 95 para la edad) y días de estancia hospitalaria, que correspondieron al tiempo total de seguimiento del paciente. Puesto que no todos los pacientes con diagnóstico de anemia requieren transfusión sanguínea se aplicaron los criterios de transfusión para anemia del prematuro recomendados por el Consenso de la Sociedad Iberoamericana de Neonatología. ${ }^{19}$

\section{Consideraciones éticas}

El estudio recibió la aprobación del Comité de Ética de la institución y se recabó el consentimiento informado de la madre o del tutor del recién nacido.

\section{Análisis estadístico}

Con el conocimiento previo de que cada año se registran en el hospital alrededor de 4000 nacimientos los participantes se asignaron al azar a una de dos opciones; $6 \%$ prematuros, con lo que se espera un promedio de 240 prematuros por año. Se consideró una proporción de 0.5 para los dos grupos de recién nacidos prematuros: pinzamiento temprano y ordeño de cordón umbilical, con intervalos de confianza de 95\% (IC95\%) y error máximo de estimación del0.054. Se utilizó la fórmula de diferencia de proporciones para un muestreo sin reemplazo con corrección para población finita: ${ }^{20}$

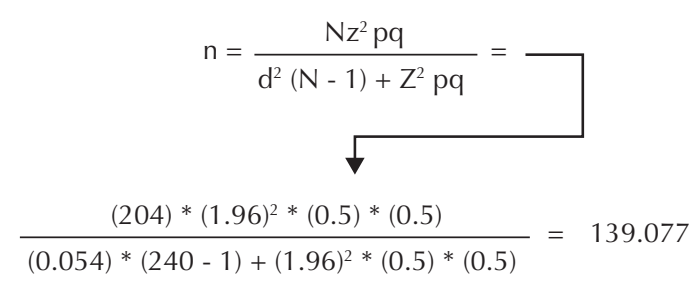

El cálculo del tamaño de muestra fue de 140 mujeres con parto prematuro, para que 70 prematuros fueran asignados 1:1 a cada grupo. Se efectuaron pruebas de normalidad a través de Kolmogorov-Smirnov, estadística descriptiva con porcentajes, medidas de tendencia central y desviación estándar. Para comparar las medias de las variables cuantitativas en los dos grupos de estudio se utilizó t de Student y para el contraste 
de las medianas de las variables cualitativas $U$ de Mann Whitney y $\chi^{2}$ para establecer independencia entre las variables categóricas. En las variables cualitativas se calculó el riesgo relativo con IC95\% como medida de relevancia clínica. También se efectúo el análisis de regresión logística múltiple con la variable dependiente requiere transfusión ajustada al peso al nacimiento, comorbilidades, hemoglobina basal y tipo de procedimiento. En todos los casos se consideró significación estadística con valor $p<0.05$. El análisis de datos se realizó en el programa estadístico SPSS versión 19.

\section{RESULTADOS}

Se seleccionaron 140 mujeres con parto prematuro de las que se excluyeron 7 por no reunir los criterios mencionados. Se asignaron al azar 134, de las que 67 se repartieron a cada grupo. Después de aplicar uno u otro procedimiento se eliminaron 21 pacientes en el grupo con ordeño y 22 en el grupo con pinzamiento temprano. El análisis final se efectúo con 91 pacientes: 46 (50.5\%) en el grupo con ordeño y 45 (49.5\%) en el grupo con pinzamiento temprano. Los criterios de selección de pacientes se muestran en la Figura 1.

En el grupo con ordeño 27 (58.7\%) pacientes fueron de sexo masculino y $23(51.2 \%)$ en el grupo con pinzamiento temprano. La edad gestacional media en el grupo con ordeño fue de $33.43 \pm 2.3$ semanas, con límites entre 27 y 36 semanas y en el grupo con pinzamiento temprano de $33.44 \pm 2.1$ semanas, con límites

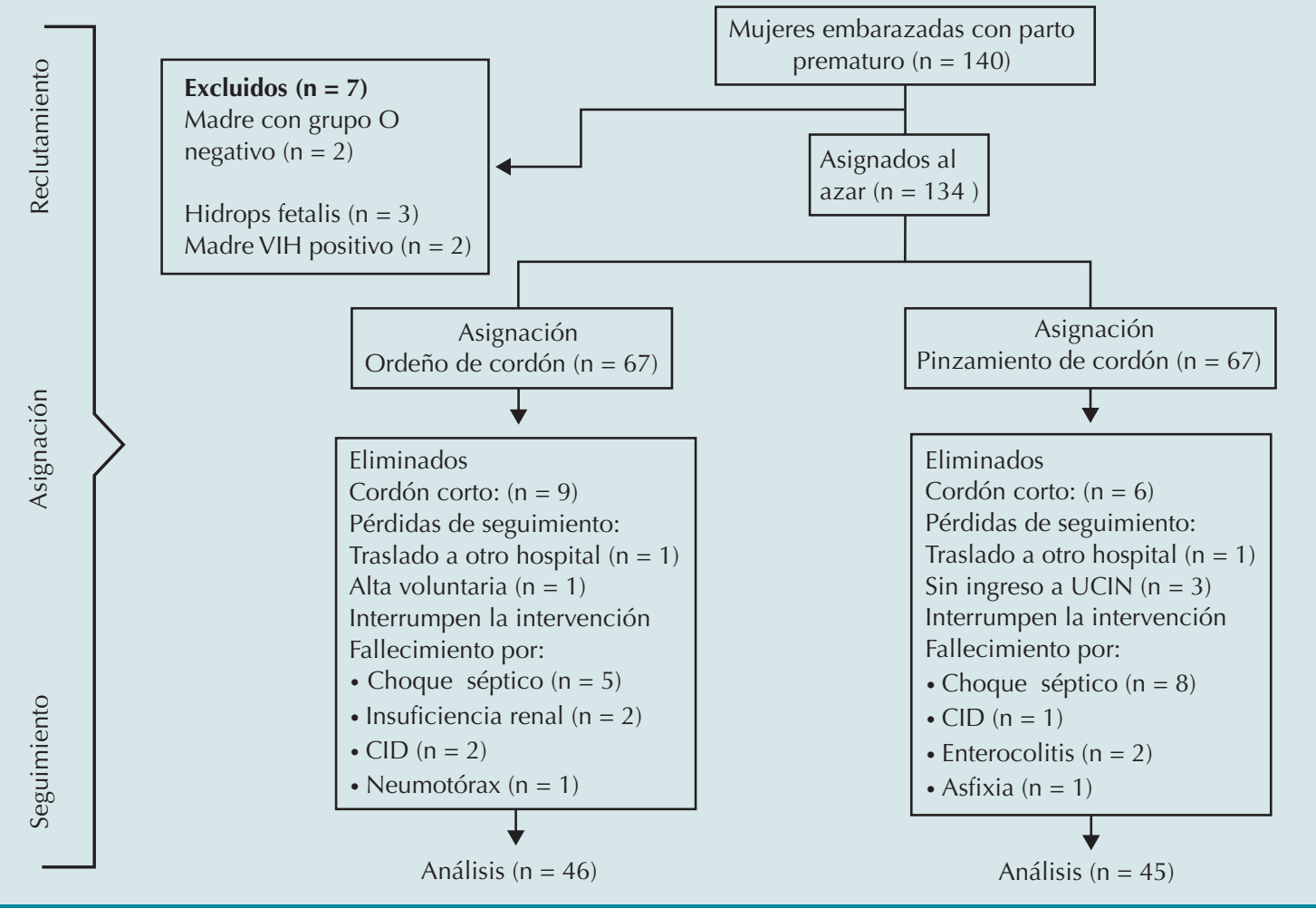

Figura 1. Selección de los pacientes 
entre 29 y 36 semanas. En el grupo con ordeño 7 pacientes tuvieron menos de 30 semanas, 15 de 30 a 33 semanas y 24 de 34 a 36 semanas, mientras que en el grupo con pinzamiento 6 pacientes tuvieron menos de 30 semanas, 14 de 30 a 33 semanas y 25 de 34 a 36 semanas.

El peso medio de los recién nacidos del grupo con ordeño fue de $1652 \pm 347$ g con límites entre 700 y 2420 g. En el grupo con pinzamiento temprano de $1663 \pm 374$ g con límites entre 735 y 2300 g. En el grupo con ordeño 6 pesaron menos de 1000 g, 13 de 1000 a 1500 g y 27 más de 1500 g. En el grupo con pinzamiento temprano, 5 tuvieron menos de 1000 g, 13 de 1000 a 1500 g y 27 más de 1500 g.

No se encontraron diferencias significativas en: sexo, peso, edad gestacional y diagnóstico principal de ingreso, entre ambos grupos. Cuadro 1

Cuadro 1. Datos generales y diagnósticos de ingreso

\begin{tabular}{|c|c|c|c|}
\hline Variable & $\begin{array}{l}\text { Ordeño } \\
(n=46)\end{array}$ & $\begin{array}{l}\text { Pinzamiento } \\
\quad(n=45)\end{array}$ & p \\
\hline \multicolumn{4}{|l|}{ Sexo } \\
\hline Masculino & $27(58.6 \%)$ & $23(51.2 \%)$ & $0.47^{*}$ \\
\hline $\begin{array}{l}\quad \text { Femenino } \\
\text { EG (semanas) } \\
\text { Peso (g) } \\
\text { SDR por déficit } \\
\text { de surfactante }\end{array}$ & $\begin{array}{c}19(41.3 \%) \\
33.43 \pm 2.3 \\
1652 \pm 374 \\
7(15.2 \%)\end{array}$ & $\begin{array}{c}22(48.8 \%) \\
33.44 \pm 2.1 \\
1663 \pm 374 \\
6(13.3 \%)\end{array}$ & $\begin{array}{l}0.33^{*} \\
0.95^{* *} \\
0.54^{* *} \\
0.80^{*}\end{array}$ \\
\hline $\begin{array}{l}\text { Neumonía } \\
\text { perinatal }\end{array}$ & $15(32.6 \%)$ & $13(28.8 \%)$ & $0.70^{*}$ \\
\hline $\begin{array}{l}\text { Riesgo de } \\
\text { sepsis por RPM }\end{array}$ & $10(21.7 \%)$ & $8(17.7 \%)$ & $0.64^{*}$ \\
\hline $\begin{array}{l}\text { Asfixia } \\
\text { perinatal }\end{array}$ & $3(6.5 \%)$ & $4(8.8 \%)$ & $0.67^{*}$ \\
\hline RCIU & $3(6.5 \%)$ & $5(11.1 \%)$ & $0.44^{*}$ \\
\hline $\begin{array}{l}\text { Taquipnea } \\
\text { transitoria }\end{array}$ & $5(10.8 \%)$ & $3(6.6 \%)$ & $0.97^{*}$ \\
\hline Hipoglucemia & $3(6.5 \%)$ & $6(13.3 \%)$ & $0.82^{*}$ \\
\hline
\end{tabular}

En el grupo con ordeño la hemoglobina basal media, tomada a las 24 horas de vida, fue de $17.08 \pm 2 \mathrm{~g} / \mathrm{dL}$ y en el grupo con pinzamiento temprano de $14.74 \pm 1.78 \mathrm{~g} / \mathrm{dL}$. Esta diferencia fue significativa $(p=0.001)$.

Se diagnosticaron con anemia 22 (47.8\%) recién nacidos del grupo con ordeño y 33 (73.3\%) del grupo con pinzamiento temprano. El riesgo relativo de anemia por pinzamiento temprano fue de 2.7 con IC95\%: 1.8-3.2, lo que resultó significativo $(p=0.001)$.

Por lo que se refiere al diagnóstico de anemia con requerimiento de transfusión sanguínea hubo 19 (41.3\%) pacientes del grupo con ordeño que requirieron alguna transfusión durante su estancia hospitalaria. En el grupo con pinzamiento temprano la requirieron 31 (68.8\%) neonatos.

El riesgo relativo de padecer anemia con requerimiento de transfusión en los recién nacidos que recibieron pinzamiento temprano fue 1.7; con IC95\%: 1.2-2.5. Esta diferencia resultó significativa $(p=0.002)$. De los pacientes que requirieron transfusiones durante el periodo de seguimiento, se encontró que en el grupo con pinzamiento temprano $9(20 \%)$ requirieron de 1 a 2 transfusiones y en el grupo con ordeño 7 (15.2\%). Del grupo con pinzamiento temprano 14 (28.8\%) recién nacidos y 8 (17.3\%) del grupo con ordeño requirieron de 3 a 5 transfusiones $y$, por último, $8(17.7 \%)$ pacientes del grupo con pinzamiento y 4 ( $8.7 \%)$ del grupo con ordeño requirieron más de 5 transfusiones sanguíneas. Con estas cifras pudo establecerse que 22 (48.8\%) recién nacidos del grupo con pinzamiento temprano requirieron más de dos trasfusiones, en comparación con $12(26.1 \%)$ del grupo con ordeño. El riesgo relativo de requerir más de dos transfusiones en pacientes con pinzamiento temprano fue de 1.9 (IC95\%:1.3-3.4), lo que resultó estadísticamente significativo $(p=0.025)$. 
El volumen promedio de transfusión en el grupo con ordeño fue de $18.2 \mathrm{~mL} \pm 33.6$ con límites entre 0 y $180 \mathrm{~mL}$. En el grupo con pinzamiento temprano de $35.3 \pm 45 \mathrm{~mL}$ con límites entre 0 y $195 \mathrm{~mL}$; diferencia estadísticamente significativa $(p=0.04)$.

En el grupo con pinzamiento temprano del cordón se identificó policitemia en 1 (2.2\%) recién nacido y en el grupo con ordeño en 2 $(4.3 \%)$, diferencia que no resultó significativa ( $p=0.57$ ) con RR e IC95\%: 0.5; 0.2-5.4. En los tres pacientes la policitemia se corrigió solo con incremento del aporte de líquidos intravenosos. En 8 (17.4\%) neonatos del grupo con ordeño se diagnosticó hiperbilirrubinemia, con una concentración máxima de bilirrubinas a las 48 horas de $14.3 \mathrm{mg} / \mathrm{dL}$ y en 7 (15.5\%) pacientes del grupo con pinzamiento temprano, con una concentración máxima de bilirrubinas de 15 mg/ dL. No se encontró una diferencia significativa en los niños con hiperbilirrubinemia ( $p=0.81)$, con RR e IC95\%: 0.9; 0.4-2.3. En todos los casos la hiperbilirrubinemia se corrigió con fototerapia, con duración promedio de 5 días.

La estancia hospitalaria media en el grupo con ordeño fue de $33 \pm 25$ días y de $37 \pm 28$ días en el grupo sin ordeño, diferencia no fue significativa $(p=0.21)$. Las características clínicas de los recién nacidos con ordeño y pinzamiento temprano se encuentran en el Cuadro 2.

Al efectuar el análisis multivariado con la variable dependiente (requerimiento de transfusión) ajustada al peso de nacimiento, edad gestacional, hemoglobina basal, comorbilidades y procedimiento se encontró que el requerimiento de transfusión está inversamente relacionado con las concentraciones de hemoglobina basal (RM e IC95\%: 0.71; 053-0.96; $p=0.02$ ).

Los recién nacidos con edad gestacional menor a 30 semanas tienen mayor riesgo de transfu-
Cuadro 2. Características clínicas de los pacientes con pinzamiento y ordeño

\begin{tabular}{|c|c|c|c|c|}
\hline Variable & $\begin{array}{c}\text { Ordeño } \\
(n=46)\end{array}$ & $\begin{array}{c}\text { Pinzamiento } \\
(\mathrm{n}=45)\end{array}$ & $\begin{array}{l}\text { RR IC } \\
95 \%\end{array}$ & $\mathbf{p}$ \\
\hline Anemia & $\begin{array}{c}22 \\
(47.8 \%)\end{array}$ & 33 (73.3\%) & $\begin{array}{c}2.7 \\
1.8-3.2\end{array}$ & $0.001 *$ \\
\hline $\begin{array}{l}\text { Requerimiento } \\
\text { de transfusión }\end{array}$ & $\begin{array}{c}19 \\
(41.3 \%)\end{array}$ & $31(68.8 \%)$ & $\begin{array}{c}1.7 \\
1.2-2.5\end{array}$ & $0.002 *$ \\
\hline $\begin{array}{l}>\text { de } 2 \\
\text { transfusiones }\end{array}$ & $\begin{array}{c}12 \\
(26.1 \%)\end{array}$ & $22(48.8 \%)$ & $\begin{array}{c}1.9 \\
1.3-3.4\end{array}$ & $0.025^{*}$ \\
\hline Policitemia & $2(4.3 \%)$ & $1(2.2 \%)$ & $\begin{array}{c}0.5 ; \\
0.2-5.4\end{array}$ & $0.57^{*}$ \\
\hline $\begin{array}{l}\text { Hiperbilirrubi- } \\
\text { nemia }\end{array}$ & $8(17.4 \%)$ & $7(15.5 \%)$ & $\begin{array}{c}0.9 ; \\
0.4-2.3\end{array}$ & $0.81^{*}$ \\
\hline
\end{tabular}

$\mathrm{RR}=$ riesgo relativo. ${ }^{*} \chi^{2}$

sión (RM e IC95\%: 5.86; 2.67-6.21; p = 0.001), al igual que los pacientes con peso menor a 1000 gramos (RM e IC95\%: 7.61; 2.52-9.36; p $=0.013)$. También se encontró que el riesgo de transfusión se incrementa a casi el doble en los pacientes que recibieron pinzamiento temprano (RM e IC95\%: 1.87; 1.05-3.31; $p=0.02$ ). Los resultados del análisis multivariado se muestran en el Cuadro 3.

\section{DISCUSIÓN}

Por lo que se refiere a los diagnósticos de ingreso se estableció que los padecimientos más frecuentes en ambos grupos fueron: neumonía perinatal y riesgo de sepsis por rotura prematura de membranas que representaron, en conjunto, $54 \%$ en pacientes con ordeño de cordón y $56 \%$ en pacientes con pinzamiento temprano. Este hallazgo puede deberse a que la mayoría de los recién nacidos pretérmino, $63 \%$ en el grupo con pinzamiento temprano y $67 \%$ en el de ordeño pesaban más 1500 g, por lo que los padecimientos propios de los recién nacidos más pequeños, como el síndrome de dificultad respiratoria por déficit de surfactante, fueron relativamente infrecuentes. 
Cuadro 3. Regresión logística múltiple para requerimiento de transfusión

\begin{tabular}{|c|c|c|c|c|}
\hline Variable & $\mathbf{N}$ & $\begin{array}{c}\text { OR } \\
\text { Ajustado }\end{array}$ & IC95\% & $\mathbf{p}$ \\
\hline \multicolumn{5}{|l|}{$\begin{array}{l}\text { Edad } \\
\text { gestacional }\end{array}$} \\
\hline $\begin{array}{c}<30 \\
30-33\end{array}$ & $\begin{array}{l}13 \\
29\end{array}$ & $\begin{array}{l}5.86 \\
1.96\end{array}$ & $\begin{array}{l}2.67-8.91 \\
1.73-6.21\end{array}$ & $\begin{array}{c}0.001 \\
0.07\end{array}$ \\
\hline \multicolumn{5}{|l|}{ Peso (gramos) } \\
\hline $\begin{array}{l}<1000 \\
1000-1500\end{array}$ & $\begin{array}{l}11 \\
26\end{array}$ & $\begin{array}{l}7.61 \\
2.34\end{array}$ & $\begin{array}{l}2.52-9.36 \\
1.12-6.80\end{array}$ & $\begin{array}{c}0.013 \\
0.56\end{array}$ \\
\hline SDR & 13 & 1.41 & $\begin{array}{l}0.90- \\
22.30\end{array}$ & 0.80 \\
\hline Neumonía & 28 & 0.52 & $0.08-3.40$ & 0.53 \\
\hline Riesgo sepsis & 18 & 0.76 & $0.11-5.12$ & 0.76 \\
\hline Asfixia & 7 & 0.26 & $0.02-3.35$ & 0.26 \\
\hline RCIU & 8 & 0.60 & $0.06-5.49$ & 0.65 \\
\hline TTRN & 8 & 0.77 & $0.08-7.13$ & 0.82 \\
\hline Hipoglucemia & 9 & 0.32 & $0.03-4.28$ & 0.76 \\
\hline Hb. basal & 91 & 0.71 & $0.53-0.96$ & 0.02 \\
\hline $\begin{array}{l}\text { Procedimiento } \\
\text { Pinzamiento } \\
\text { temprano }\end{array}$ & 45 & 1.87 & $1.05-3.31$ & 0.02 \\
\hline
\end{tabular}

Ajustado por: SDR: síndrome de dificultad respiratoria por déficit de surfactante, RCIU: restricción de crecimiento intrauterino

TTRN: taquipnea transitoria del recién nacido, $\mathrm{Hb}$. basal: hemoglobina basal.

Las concentraciones de hemoglobina a las 24 horas de vida fueron significativamente mayores en los recién nacidos con ordeño de cordón, lo que es semejante a los hallazgos de March y su grupo $^{21}$ en Estados Unidos en recién nacidos extremadamente prematuros. Si bien en el estudio de este grupo las concentraciones de hemoglobina fueron más bajas en ambos grupos $(14.9 \mathrm{~g} / \mathrm{dL}$ en el grupo con ordeño vs $13.6 \mathrm{~g} / \mathrm{dL}$ en el grupo con pinzamiento temprano) en relación al aquí reportado $(17.08 \mathrm{~g} / \mathrm{dL}$ en el grupo con ordeño vs $14.74 \mathrm{~g} / \mathrm{dL}$ en el grupo con pinzamiento temprano) lo que puede estar asociado con la altitud sobre el nivel del mar de la Ciudad de México, que condiciona mayor grado de policitemia relativa en los recién nacidos..$^{22,23}$

El estudio efectuado por Erickson-Owens ${ }^{24}$ en recién nacidos a término también reportó concentraciones más elevadas de hemoglobina entre las 36 y 48 horas en los recién nacidos con ordeño en relación con los de pinzamiento temprano: $19 \pm 2.1$ vs $17.2 \pm 2.1 \mathrm{~g} / \mathrm{dL}$, lo que se explica por la transferencia activa de sangre del cordón en dirección al recién nacido porque con este procedimiento es posible transferir entre 30 y $40 \mathrm{~mL}$ de sangre.

Los pacientes que recibieron pinzamiento temprano del cordón tuvieron más del doble de riesgo de padecer anemia en relación con quienes recibieron ordeño de cordón. En este último grupo se registró una reducción de $25 \%$ en la frecuencia de esta afección. De la misma manera, los pacientes que recibieron ordeño de cordón tuvieron una reducción de la necesidad de transfusión sanguínea de $27.5 \%$ en relación con quienes solo se efectúo el pinzamiento temprano del cordón en los que el riesgo de requerir una transfusión sanguínea se elevó a casi el doble.

Por lo que se refiere a la cantidad de transfusiones recibidas pudo establecerse que los pacientes con ordeño del cordón recibieron más de 2 transfusiones en forma significativamente mayor que el grupo que recibió pinzamiento temprano. Esto es evidente porque solo 26.1\% de lo pacientes con ordeño de cordón requirieron más de 2 transfusiones en comparación con $48.8 \%$ de los recién nacidos con pinzamiento temprano. Esta reducción en la necesidad de transfusión de glóbulos rojos también fue descrita por Hosono y su grupo ${ }^{25}$ con cifras muy similares a las encontradas en nuestro estudio.

De acuerdo con los resultados comentados, también se encontró una reducción considerable en el volumen promedio de sangre trasfundida 
entre uno y otro grupo: $18.2 \mathrm{~mL}$ en el grupo con ordeño y $35.3 \mathrm{~mL}$ en el grupo con pinzamiento temprano, lo que también hace evidente una reducción de la necesidad de transfusión sanguínea en el grupo con ordeño.

Nuestros resultados corroboran la utilidad del ordeño del cordón en la prevención de anemia, reducción del requerimiento de transfusiones sanguíneas, de la cantidad de transfusiones y del volumen de sangre transfundida, lo que a su vez puede ser benéfico para los recién nacidos al incrementar los depósitos de hierro desde el nacimiento hasta los 6 meses de edad, como lo describen Bora $\mathrm{R}$ y sus colaboradores. ${ }^{26}$

La transfusión de glóbulos rojos, que es el procedimiento más utilizado para tratar la anemia, puede ocasionar una serie de complicaciones en el recién nacido: trasmisión de infecciones, alteraciones en la inmunomodulación, enfermedad de injerto contra huésped, lesión pulmonar aguda, aloinmunización, disturbios electrolíticos, incompatibilidad sanguínea y mayor riesgo de enterocolitis necrosante. ${ }^{27}$ Por todo esto la transfusión de glóbulos rojos es un tratamiento temporal e imperfecto, lo que recalca la importancia de implementar procedimientos, como el ordeño del cordón umbilical, que reduzcan la necesidad de transfusiones sanguíneas.

Si bien la incidencia de policitemia e hiperbilirrubinemia fue ligeramente superior en el grupo de pacientes con ordeño, no hubo una diferencia significativa entre ambos grupos y esas alteraciones fueron leves porque se corrigieron con procedimientos sencillos, como el incremento del aporte de líquidos intravenosos y la fototerapia. Estos hallazgos son similares a los encontrados por March y su grupo ${ }^{21}$ en recién nacidos pretérmino y Erickson-Owens ${ }^{24}$ y Yadav y colaboradores ${ }^{28}$ en recién nacidos a término. Con base en ellos, la policitemia y la ictericia no contraindican el ordeño de cordón, sobre todo tomando en cuenta que son leves, se pueden tratar fácilmente en centros hospitalarios provistos del equipo y la tecnología necesarios, que es donde habitualmente nacen estos pacientes..$^{22,23}$

Pese a que en el grupo de pacientes con ordeño se identificó una reducción del tiempo de estancia hospitalaria de 4 días en promedio, ésta no fue significativa, lo que puede deberse a que esta variable en el recién nacido pretérmino es influida, además de la anemia, por múltiples factores: grado de prematurez, padecimientos asociados durante la estancia hospitalaria, equipamiento hospitalario, dotación de insumos y complicaciones propias de este grupo de pacientes. La tendencia al egreso más temprano de estos recién nacidos puede ser otro factor importante que considerar para estimular la práctica de este procedimiento.

Al analizar los factores que contribuyen directamente a la necesidad de transfusión sanguínea se estableció que la prematurez extrema fue la causa base más frecuente de anemia y requerimiento de transfusión. En los recién nacidos extremadamente prematuros la necesidad de transfusión fue mayor, independientemente del procedimiento utilizado. Por esto, el ordeño del cordón tuvo efectos más evidentes en los recién nacidos mayores de 30 semanas y con peso mayor de 1000 g, que es la población más numerosa que se atiende en nuestro hospital.

De la misma manera, las concentraciones de hemoglobina basal más elevadas, propias de los recién nacidos con ordeño del cordón, son un factor que reduce la cantidad de transfusiones sanguíneas y, por el contrario, el pinzamiento temprano como procedimiento inicial incrementa directamente la necesidad de transfusiones. Estos resultados son muy similares a los encontrados por Hosono y su grupo ${ }^{25}$ en Japón, aunque en ese estudio el ordeño de cordón también redujo significativamente la necesidad de 
trasfusiones en recién nacidos extremadamente prematuros.

Una limitante de este trabajo fue que no se estudió el efecto del ordeño del cordón en la evolución de otras enfermedades frecuentes en los recién nacidos prematuros: hemorragia intraventricular, enterocolitis necrosante, retinopatía del prematuro y displasia broncopulmonar. Esta investigación sigue aún en curso en nuestra institución.

\section{CONCLUSIONES}

El ordeño del cordón umbilical es un procedimiento sencillo y eficaz para prevenir, en recién nacidos prematuros, la anemia y reducir el requerimiento de transfusiones sanguíneas derivadas de este padecimiento. En recién nacidos con anemia, pese al ordeño del cordón existe, también, menor necesidad de transfusiones y, por el contrario, la práctica habitual del pinzamiento temprano del cordón incrementa a casi el doble el riesgo de requerir transfusiones sanguíneas, por lo que debe considerarse la necesidad de modificar esta práctica aún habitual en muchos centros hospitalarios.

Además, es importante insistir que esta técnica de trasferencia activa de sangre al recién nacido no se asocia con reacciones adversas severas, por eso puede catalogarse como un procedimiento seguro, con la ventaja de no interferir con la reanimación neonatal en prematuros críticamente enfermos, como sucede con el pinzamiento tardío del cordón umbilical.

Aunque en nuestro estudio hubo una tendencia al egreso hospitalario más temprano en los recién nacidos con ordeño del cordón, ésta es una variable dependiente de muchos otros factores, por lo que consideramos que debe tomarse con cautela. Al ser un procedimiento con potenciales efectos benéficos en la evolución de los recién nacidos pretérmino debe estudiarse la posibilidad de implementarlo como un procedimiento de rutina en la atención inicial de estos pacientes.

\section{REFERENCIAS}

1. Bishara N, Ohls R. Current controversies in the management of the anemia of prematurity. Semin Perinatol. 2009;33:2934. https://doi.org/10.1053/j.semperi.2008.10.006

2. Aher S, Malnather K. Kadam S. Neonatal anemia. Semin Fetal Neonatal Med. 2008;13:239-47.

3. Widness JA. Treatment and Prevention of Neonatal Anemia. Neoreviews 2008; 9(11): 526-533.

4. Arca G, Carbonell-Estrany XB. Anemia neonatal. Protocolos Diagnósticos Terapéuticos de la AEP: Neonatología, 2008.

5. Lemus-Varela L, Sola A. Manual práctico para toma de decisiones en hematología neonatal. $1^{\mathrm{a}} \mathrm{ed}$. Consenso SIBEN, Buenos Aires, 2011.

6. Luban NLC. Management of anemia in the newborn. Early Hum Dev. 2008;84: 493-98. https://doi.org/10.1016/j. earlhumdev.2008.06.007

7. Figueras JA. Eritropoyetina en Neonatología. An Pediatr (Barc). 2010;73(6):301-4. doi: 10.1016/j.anpedi.2010.09.004.

8. Rosenberg J. Umbilical cord milking for anemia prevention. From opposing view points in context. IPSRH. 2013;39:57.

9. Katheria A, et al. A new wrinkle: Umbilical cord management (how, when, who). Seminars in Fetal and Neonatal Medicine (2018), https://doi.org/10.1016/j. siny.2018.07.003

10. Hosono S, et al. One-time umbilical cord milking after cord cutting has some effectiveness as multiple-time umbilical cord milking in infants born at $<29$ weeks of gestation: a retrospective study. J Perinatol. 2015;35:590-94.

11. Patel $\mathrm{S}$, et al. Effects of umbilical cord milking on morbidity and survival in extremely low gestational age neonates Am J Obstet and Gynecol. 2014;211:519-27. https://doi. org/10.1016/j.ajog.2014.05.037

12. Al-Wassia H, Shah PS. Efficacy and Safety of Umbilical Cord Milking al Birth: A systematic Review and Meta-analyses. JAMA Pediatr. 2015;169:18-25. doi:10.1001/jamapediatrics.2014.1906

13. Upadhyay A, et al. Effect of umbilical cord milking in term and near term infants: randomized control trial. Obst Gynecol Surv. 2013; 68:333-34. https://doi.org/10.1016/j. ajog.2012.10.884

14. Jaiswal $\mathrm{P}$, et al. Comparison of umbilical cord milking and delayed cord clamping in term neonates: A randomized controlled trial. AJPN. 2015;1:1-7. https://doi. org/10.1007/s12098-015-1734-2 
15. Katheria AC, Truong G, Counsins L. Umbilical Cord Milking versus Delayed Cord Clamping in preterm infants. Pediatrics. 2015;136(1):61-69. doi: 10.1542/peds.2015-0368

16. Mercer JJ. Current best evidence: a review of the literature on umbilical cord clamping. J.Midwiferry Women Health 2001;46:402-14. https://doi.org/10.1016/S15269523(01)00196-9

17. Uwins C. Hutchon D Jr. Delayed umbilical cord clamping after childbirth: potential benefit to baby's health. Pediatric Health, Medicine and Therapeutics 2014; 5: 16171. https://doi.org/10.2147/PHMT.S51867

18. Golsmit G, Castellanos SOR, Lacarruba J, Sposito S. Diagnóstico y Tratamiento de la anemia neonatal precoz. En: Golombek S, Lemus Varela M. Sola A. Editores. Manual Práctico para toma de decisiones en hematología Neonatal. Consenso Sociedad Iberoamericana de Neonatología. $1^{\text {a }}$ ed. Buenos Aires: Edimed, 2011:11-14.

19. Lima V, Natta D, Mir VR. Hemotransfusiones. En Golombek S, Lemus Varela M. Sola A. Editores. Manual Práctico para toma de decisiones en hematología Neonatal. Consenso. Sociedad Iberoamericana de Neonatología. $1^{\text {a }}$ ed. Buenos Aires: Edimed, 2011:36-38.

20. Wayne W. Bioestadística: Base para el Análisis de las Ciencias de la Salud. México: Limusa,1980; 141.

21. March MI, et al.The effects of umbilical cord milking in extremely preterm infants: a randomized controlled trial. J Perinatol. 2013;33:763-67.
22. López SN. La biometría hemática. Acta Pediatr Mex. 2016; 37(4):246-49.

23. Consejo de Salubridad General-México. Diagnóstico y Tratamiento de Policitemia Neonatal en el segundo y tercer nivel de atención. Catálogo Maestro de Guías de Práctica Clínica: IMSS-782-15.

24. Erickson-Owens DA, Mercer JS, Oh W. Umbilical cord milking in term infants delivery by caesarean section: a randomized controlled trial. J Perinatol. 2012;32: 580-584.

25. Hosono $\mathrm{S}$, et al. Umbilical cord milking reduces the need for red cell transfusions and improves neonatal adaptation in infants born at less than 29 weeks gestation: a randomized controlled trial . Arch Dis Child Fet Neonatal Ed. 2008;93:14-19. http://dx.doi.org/10.1136/ adc.2006.108902

26. Bora R, et al. Effect of 40-cm segment umbilical cord milking on hemoglobin and serum ferritin at 6 months of age in full term infants of anemic and non anemic mothers. J Perinatol. 2015;35:832-36.

27. Whyte R, Jeffenes A. Red Blood Cell Transfusions in Newborns Infants. Paediatr Child Health. 2014;19(4):213-17.

28. Yadav AK, et al. Comparation of three types of intervention to enhance placental redistribution in terms newborns: Randomized Control Trial. J Perinatol. 2015;35 (9):720-24. 